\title{
Adverse haemodynamic effects of serious thoracic injuries during CPR: a study based on the Campbell diagram.
}

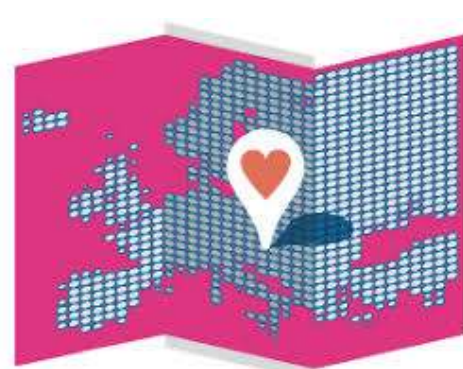

Authors: Youcef Azeli¹,2,3, Juan Víctor Lorente Olazabal ${ }^{4,5}$, Manuel Ignacio Monge García ${ }^{6}$, Alfredo Bardaji

1. Emergency Department, Sant Joan University Hospital. Reus. 2. Sistema d'Emergències Mèdiques de Catalunya.

3. IISPV 4. Clinical Management Anaesthesiology Unit, Resuscitation and Pain Therapy. Juan Ramón Jiménez Hospital. Huelva. 5. School of Medicine and Health Sciences, International University of Catalonia (IUC). 6. Intensive Care Department, University Hospital of SAS Jerez. Jerez de la Frontera. 7. Cardiology Department, Joan XXIII University Hospital. Tarragona. 8.School of Medicine, Rovira i Virgili University, Tarragona. Spain.

*youcefazeli@gencat.cat

Purpose of the Study: Serious thoracic injuries (STIs) are common during cardiopulmonary resuscitation (CPR). The aim of this study was to establish the effect of STIs on haemodynamics during CPR using a theoretical approach based on the Campbell diagram (CD).

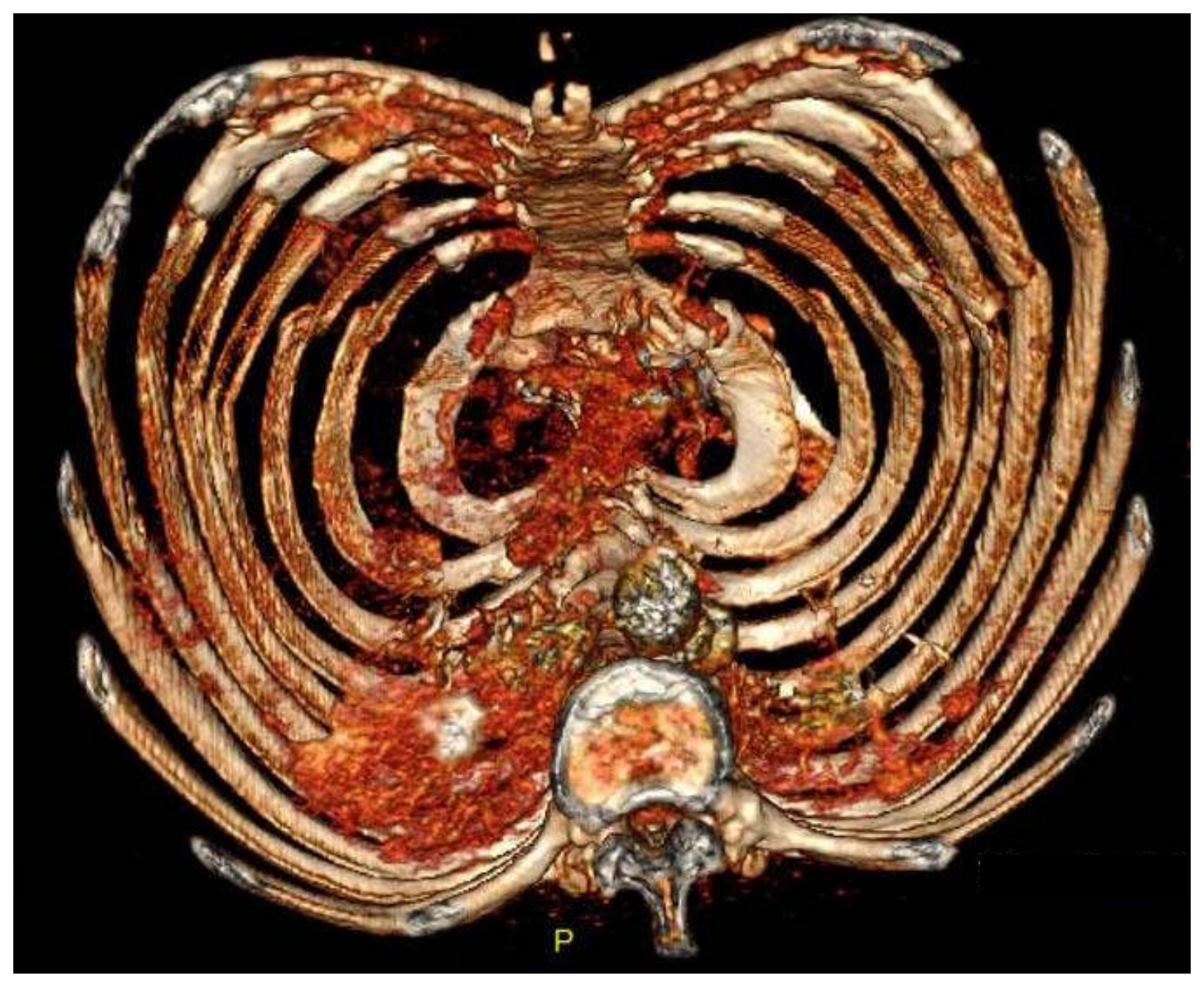

CT of a 69 years man after 40 min of manual and mechanical CPR. Source: Institute of Legal Medicine,Catalonia,

Material and methods: A CD is a theoretical framework of the respiratory system that integrates pressure-volume curve of the lung $\left[\mathrm{P}_{\mathrm{el}(\mathrm{L})}\right]$ and pressure-volume curve of the chest wall $\left[\mathrm{P}_{\mathrm{el}(\mathrm{cw})}\right]$. The point where the curves cross corresponds to the functional residual volume (FRV). It permits to represent the thorax in the decompression phase when there is no ventilation during CPR. The authors reviewed the literature that provides information on the construction of the $C D$ and the assessment of the haemodynamic effect of STIs during CPR.
Results: Multiple rib fractures on one side ${ }^{1}$ and intrathoracic visceral injuries on the other side ${ }^{2}$ has been associated with a decrease on respiratory system compliance. When representing the fall in compliance in both pressure-volume curve of the $C D$, a new balance point is established with a new, lower FRV and a higher pleural pressure (fig). An increase in pleural pressure in the chest decompression phase contributes to reduce the gradient between the mean systemic filling pressure and the right atrium pressure impairing the venous return flow. Multiple rib fractures were associated with a worse thoracic recoil and significantly less coronary perfusion ${ }^{3}$.

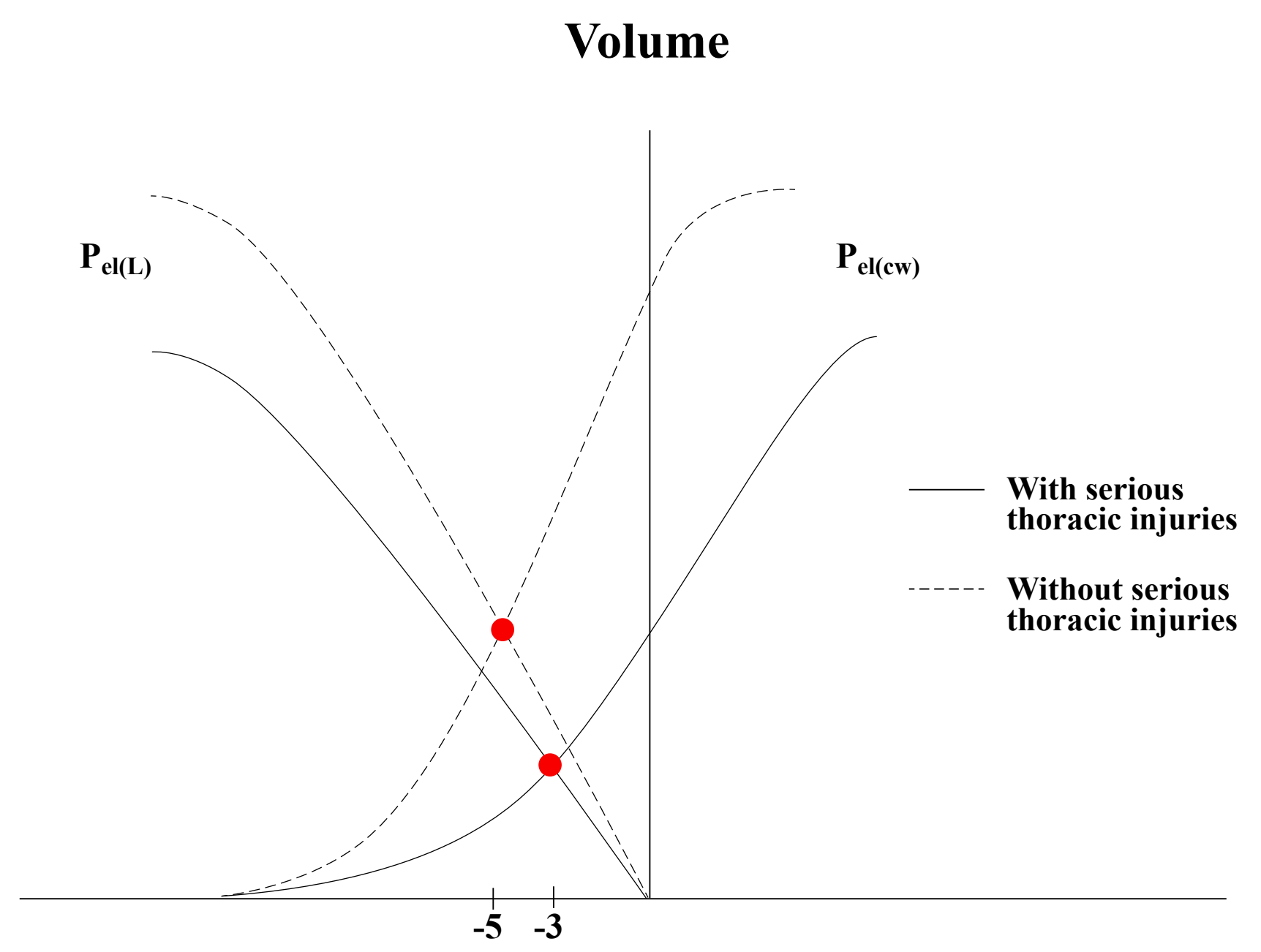

Pleural pressure

Conclusions: The Campbell diagram is a useful tool to represent the thorax biomechanics with STIs and its adverse hemodynamic effects during CPR. 